Supplementary Information for Manuscript titled "An electrocatalytic reaction as a basis for chemical computing in water droplets" by Zhijie Yang, ${ }^{\dagger,+,}$ Qiang Zhuang, ${ }^{\dagger}, \|$ Yong Yan $^{\Delta}$, Guillermo Ahumada ${ }^{\dagger}$, Bartosz A. Grzybowski, ${ }^{\dagger},{ }^{*}$

${ }^{\dagger}$ Center for Soft and Living Matter of Korea's Institute for Basic Science (IBS), Ulsan, 49919, South Korea.

‡ School of Chemistry and Chemical Engineering, MOE Key Laboratory of Colloid and Interface Chemistry, Shandong University, Jinan, 250100, China.

\| School of Chemistry and Chemical Engineering, Northwestern Polytechnical University, Xi'an, 710000, China.

$\triangle$ CAS Key Laboratory of Nanosystem and Hierarchical Fabrication, CAS Center for Excellence in Nanoscience, National Center for Nanoscience and Technology, Beijing 100190, China.

${ }^{\S}$ Department of Chemistry, Ulsan National Institute of Science and Technology, Ulsan, 49919, South Korea.

II These authors contributed equally.

* Correspondence to B.A.G. at nanogrzybowski@gmail.com

\title{
Methods
}

Synthesis of $\mathbf{A u}-\mathrm{Fe}_{3} \mathrm{O}_{4} \boldsymbol{J N P}$. Seeded growth method was used to synthesize $\mathrm{Au}-\mathrm{Fe}_{3} \mathrm{O}_{4} \mathrm{JNPs}$. $\mathrm{Au}$ nanoparticle seeds of $\sim 6 \mathrm{~nm}$ in diameter were synthesized according to our previous report with some modifications (Wu et. al. Nano Res. 2009, 2, 975-983.). 2 mmol of tertbutylamine-borane complex (97\%, Strem) was dissolved in toluene $(2 \mathrm{~mL})$ and oleylamine (70\%, Sigma-Aldrich, $2 \mathrm{~mL}$ ) by sonication. This reducing solution was rapidly injected into a precursor solution containing toluene $(10 \mathrm{~mL})$, oleylamine $(10 \mathrm{~mL})$, and $\mathrm{HAuCl}_{4} \cdot 3 \mathrm{H}_{2} \mathrm{O}(>99.9 \%$, Sigma-Aldrich, $200 \mathrm{mg})$ under a nitrogen atmosphere at room temperature $\left(\sim 23^{\circ} \mathrm{C}\right)$. The reduction occurred instantaneously, as indicated by a change in color from orange to purple-red. The reaction was left stirring under the nitrogen atmosphere for $30 \mathrm{~min}$ before ethanol $(50 \mathrm{~mL})$ was added to precipitate the $\mathrm{Au}$ nanoparticles. The Au nanoparticles ( $\sim \mathrm{nm}$ in diameter) were collected by centrifugation (6000 r.p.m., $5 \mathrm{~min}$ ), purified by three rounds of precipitation in ethanol $(20 \mathrm{~mL})$, and redispersed in hexane, giving a concentration of Au about $10 \mathrm{mg} / \mathrm{mL}$. An iron precursor solution containing iron(III) acetylacetonate (97\%, Sigma-Aldrich, $280 \mathrm{mg}$ ), oleic acid (90\%, Sigma-Aldrich, $10.8 \mathrm{~mL}$ ), and oleylamine (70\%, Sigma-Aldrich, $16 \mathrm{~mL}$ ) in a 100 $\mathrm{mL}$ three-neck flask was heated to $180{ }^{\circ} \mathrm{C}$ within $30 \mathrm{~min}$ and left stirring at $180{ }^{\circ} \mathrm{C}$ for 10 min under argon flow. A seed solution of $20 \mathrm{mg}$ Au nanoparticles in hexane $(0.5 \mathrm{~mL})$ and oleylamine $(1.5 \mathrm{~mL})$ was injected into the flask, followed by the addition of oleic acid (4 $\mathrm{mL}$ ). $180 \mathrm{~min}$ after injection, the solution was further heated to $300{ }^{\circ} \mathrm{C}$. The reaction was 
kept at $300{ }^{\circ} \mathrm{C}$ for $30 \mathrm{~min}$. At the end of the synthesis, the flask was cooled down to room temperature, and nanoparticles were purified by three rounds of precipitation in acetone $(30 \mathrm{~mL})$, and centrifugation (7000 r.p.m., $10 \mathrm{~min}$ ) with dissolution in toluene after each step. Finally, nanoparticles comprising $\sim 6 \mathrm{~nm} \mathrm{Au}$ domains and $\sim 12 \mathrm{~nm} \mathrm{Fe}_{3} \mathrm{O}_{4}$ domains were dissolved in 1,2-dichorobenzene (DCB, 99\%, Sigma-Aldrich) at a concentration of $\sim 4 \mathrm{mg} / \mathrm{mL}$.

Preparation of macroscopic droplets covered by $\mathbf{A u}-\mathrm{Fe}_{3} \mathrm{O}_{4} \mathrm{JNP}$. Ligand exchange on the JNPs' Au domains with 11-mercaptoundecanoic acid (MUA, 95\%, Sigma-Aldrich) or 3mercaptopropionic acid (MPA, 99\%, Sigma-Aldrich) was performed first. In a typical experiment, MUA or MPA (20 mg) was dissolved in DCB (3 mL) in a $15 \mathrm{~mL}$ conical, polyethylene tube to form a clear solution, followed by the addition of $50 \mu \mathrm{L}$ of JNP solution. The mixture was agitated by a vortex mixer (3000 r. p. m., $2 \mathrm{~min}$ ). $1 \mathrm{~mL}$ of an aqueous solution containing $1 \mathrm{mM} \mathrm{HCl}$ was added into the JNP mixture. The as-formed oilwater emulsion was agitated by vortex mixer for another $2 \min$ (3000 r.p.m.) to ultimately yield aqueous droplets with an average diameter of $\sim 2 \mathrm{~mm}$ in the upper phase.

Electrochemical measurements of $\mathbf{A u - F _ { 3 } \mathrm { O } _ { 4 }} \mathbf{J N P}$. HER electrochemical measurements were carried out on an electrochemical workstation (PARSTAT MC multichannel potentiostat) using a standard three-electrode setup. The $\mathrm{Ag} / \mathrm{AgCl}$ (in $3 \mathrm{M} \mathrm{NaCl}$ solution) electrode and glassy carbon (GC) rotating disk electrode (RDE) were used as a reference and working electrode, respectively. A graphite rod was used as a counter electrode. The GC RDE was polished using alumina suspensions. The working electrode $(3 \mathrm{~mm}$ in diameter) was prepared by deposition of a $\sim 2 \mathrm{~mm}$ droplet covered by MUA-Au/ $\mathrm{Fe}_{3} \mathrm{O}_{4}-\mathrm{OA}$ JNP, followed by the removal of the solvent under reduced pressure ( $\sim 10$ mbar). HER polarization curves were recorded in nitrogen-saturated deionized water or $\mathrm{HCl}$ aqueous solution $(10 \mathrm{mM})$ at a scan rate of $2 \mathrm{mV} / \mathrm{s}$ and a $1600 \mathrm{r}$. p. m. RDE. Control experiment for bare GC electrode without the loading of JNP was carried out in $\mathrm{HCl}$ aqueous solution (10 $\mathrm{mM}$ ) at a scan rate of $2 \mathrm{mV} / \mathrm{s}$ and a $1600 \mathrm{r}$. p. m. RDE.

Droplet based electronics. An external potential source, Keithley 4200, was used for device characterization. High-performance Probe Station with multiple micromanipulators was used to interface probe tips (gold) with the droplet(s).

Single droplet diode: One droplet covered with JNPs was suspended in DCB in a PTFE Petri dish. Two gold probe electrodes were connecting the droplet: One was placed touching the external surface of the droplet, the other was penetrating into the droplet. The potential was applied to the electrode touching with JNP membrane and was swept between $-2 \mathrm{~V}$ and $+2 \mathrm{~V}$. Different scan rates, such as 0.03 and $0.67 \mathrm{~V} / \mathrm{s}$, were applied during the 
measurements.

Droplet rectifiers: A half-wave rectifier was fabricated by using a single droplet. A fullwave rectifier was fabricated by using four droplets wired as illustrated in main-text Fig. 2b.

Three-electrode architecture: One droplet covered with JNP was suspended in DCB in a PTFE Petri dish. Three gold probe electrodes were connecting the droplet: two were placed touching the external surface of the droplet, the other was penetrating into the droplet. Potential was applied to the inner electrode $(0,0.5,1.0,1.5$ and $2.0 \mathrm{~V})$. The bias potential was applied between the outer electrodes and was swept between $-2 \mathrm{~V}$ and $+2 \mathrm{~V}$ with a scan rate of $0.03 \mathrm{~V} / \mathrm{s}$.

Droplet-based logic gates and inverters: "AND" or "OR" logic gates were fabricated using three droplets, of which two were covered by JNP nanosurfactants and the third was an uncoated droplet of $\mathrm{NaCl}$ solution. In the inverter, only one JNP-coated droplet was used.

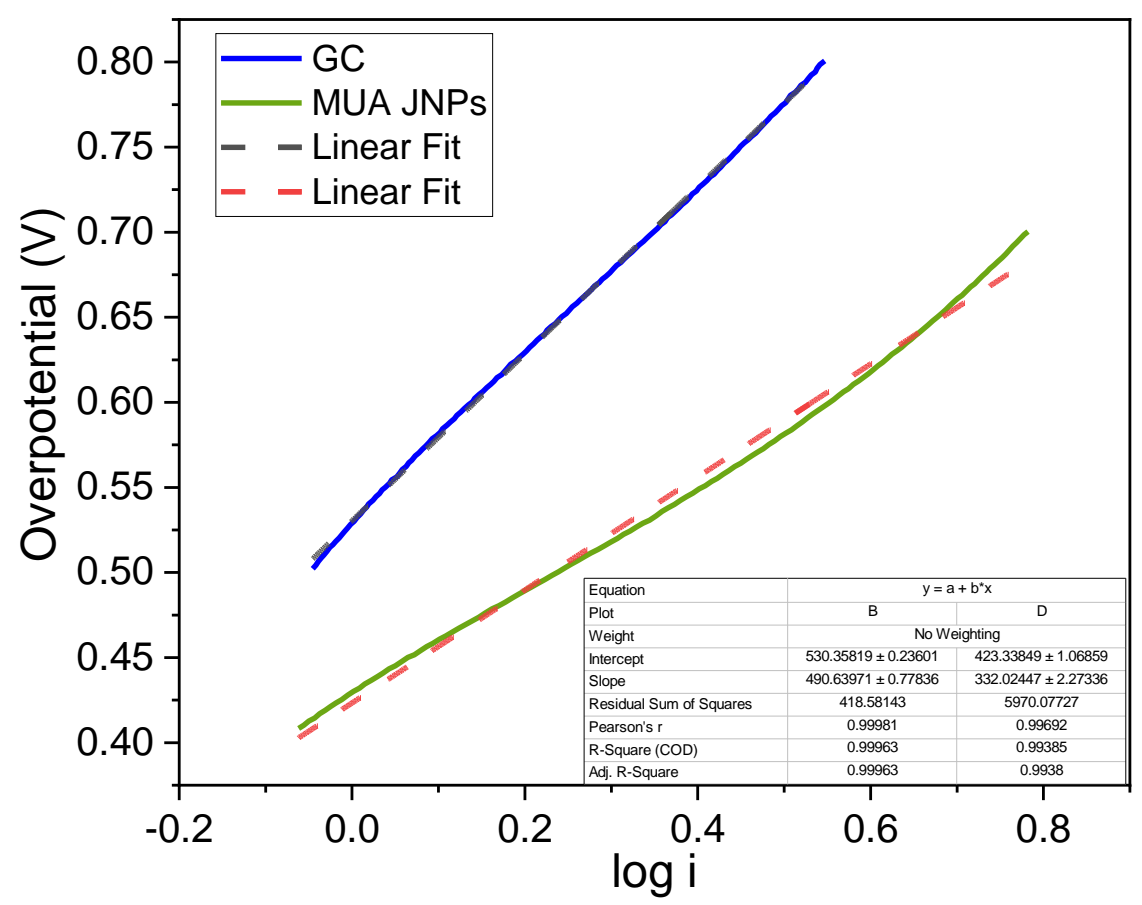

Fig. S1. Tafel plots of bare glassy carbon and MUA JNPs membrane deposited on glassy carbon (cf. main-text Fig. 1b). The values of Tafel slopes were $490 \mathrm{mV} / \mathrm{dec}^{-1}$ for bare glassy carbon electrode and $332 \mathrm{mV} / \mathrm{dec}^{-1}$ for the electrode covered with MUA JNPs. The decreased value for the latter indicates increased HER activity. 


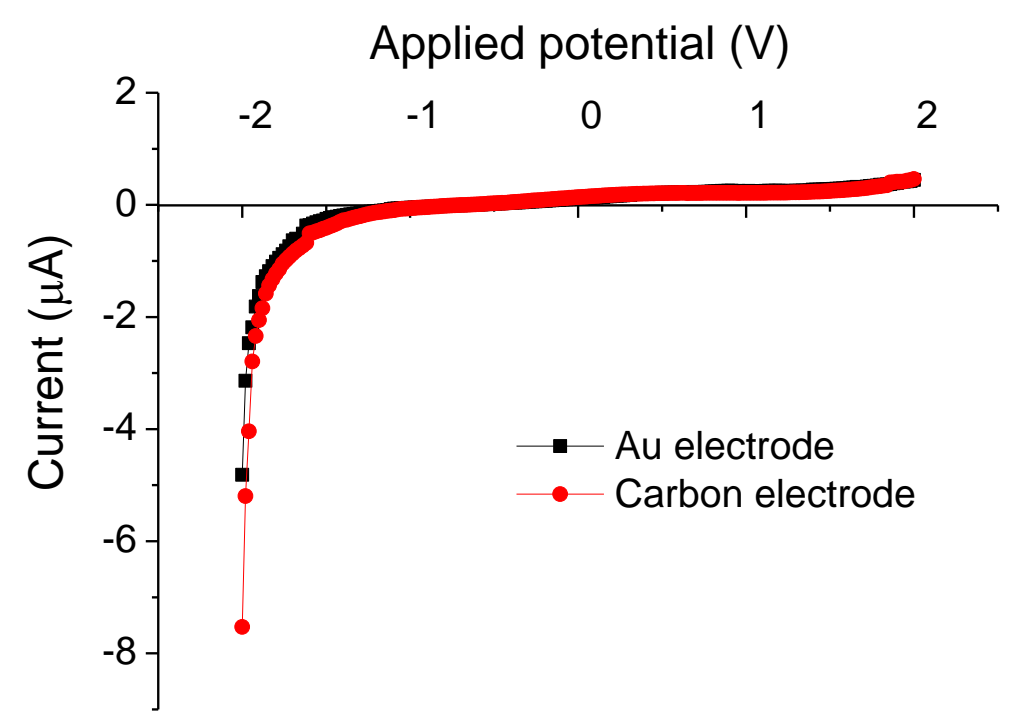

Fig. S2. Current-voltage characteristics of MUA JNPs droplets contacted by Au vs. carbon electrodes. Sweep rates were $0.1 \mathrm{~V} / \mathrm{s}$ in both cases.
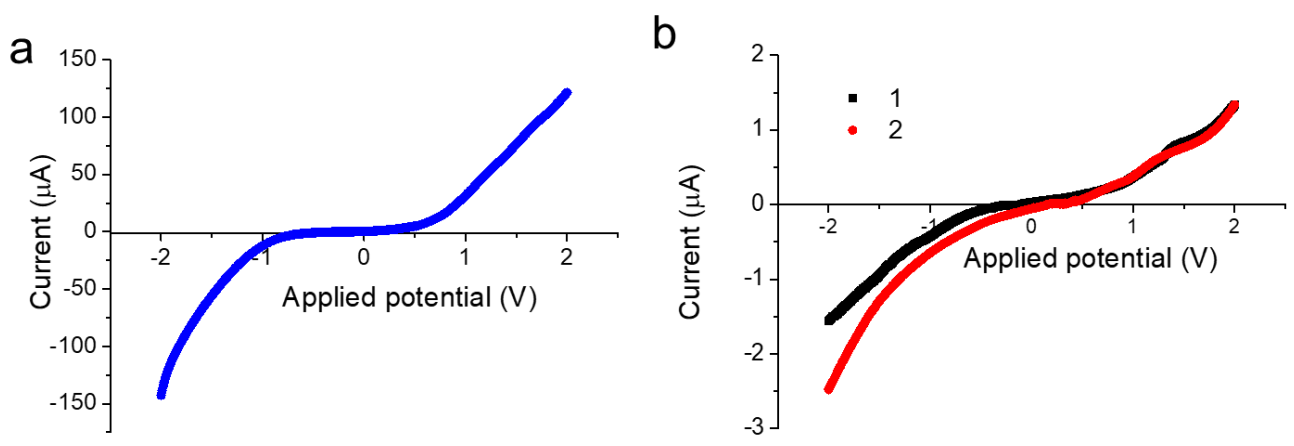

Fig. S3. Typical $I-V$ curves of two electrodes at different regions of JNP-coated droplets: (a) both electrodes were penetrating into the droplet; and (b) both electrodes were touching the external surface of the droplet. In (b) two independent experiments were conducted, and the difference can be caused by the unequal area touched by the electrodes.

a

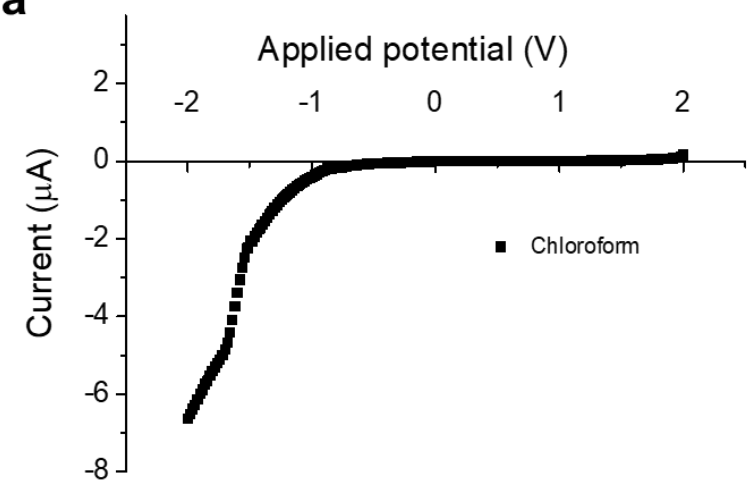

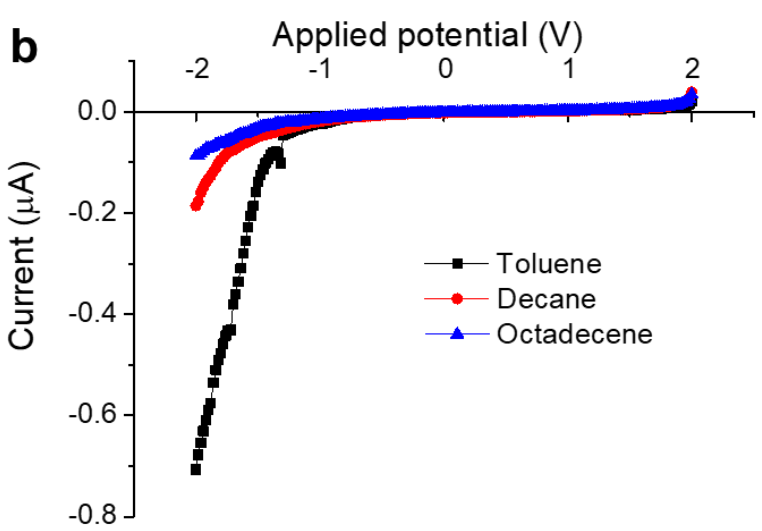

Fig. S4. (a) Current-voltage characteristics of MUA JNPs droplets in (a) chloroform and (b) toluene, decane, and octadecene. Sweep rates were $0.1 \mathrm{~V} / \mathrm{s}$ in all cases. Rectification 
ratios $r=\left|I_{-2 \mathrm{~V}} / I_{+2 \mathrm{~V}}\right|$ were 16 for chloroform, 18 for toluene, 4.5 for decane, and 2.4 for octadecene.

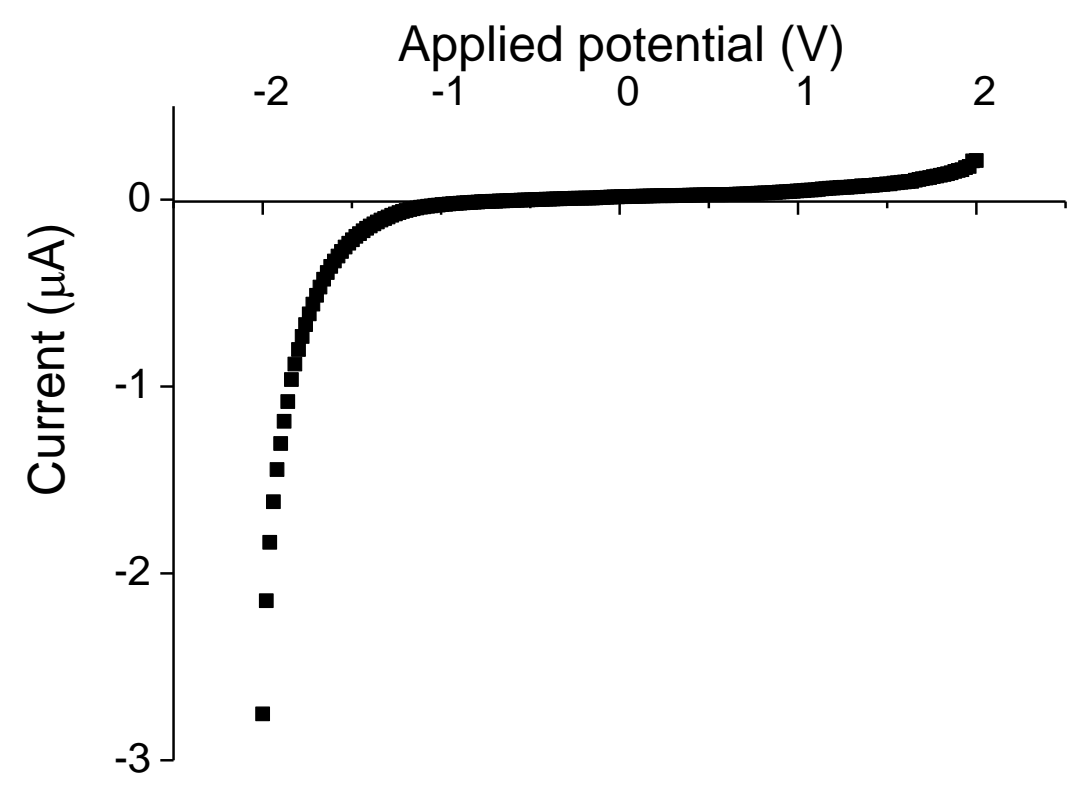

Fig. S5. Current-voltage characteristics of a MUA JNPs droplet carrying $1 \mathrm{mM} \mathrm{HNO}$ instead of $1 \mathrm{mM} \mathrm{HCl}$. The rectification ratio is $\sim 6.6$. The sweep rate used was $0.1 \mathrm{~V} / \mathrm{s}$.
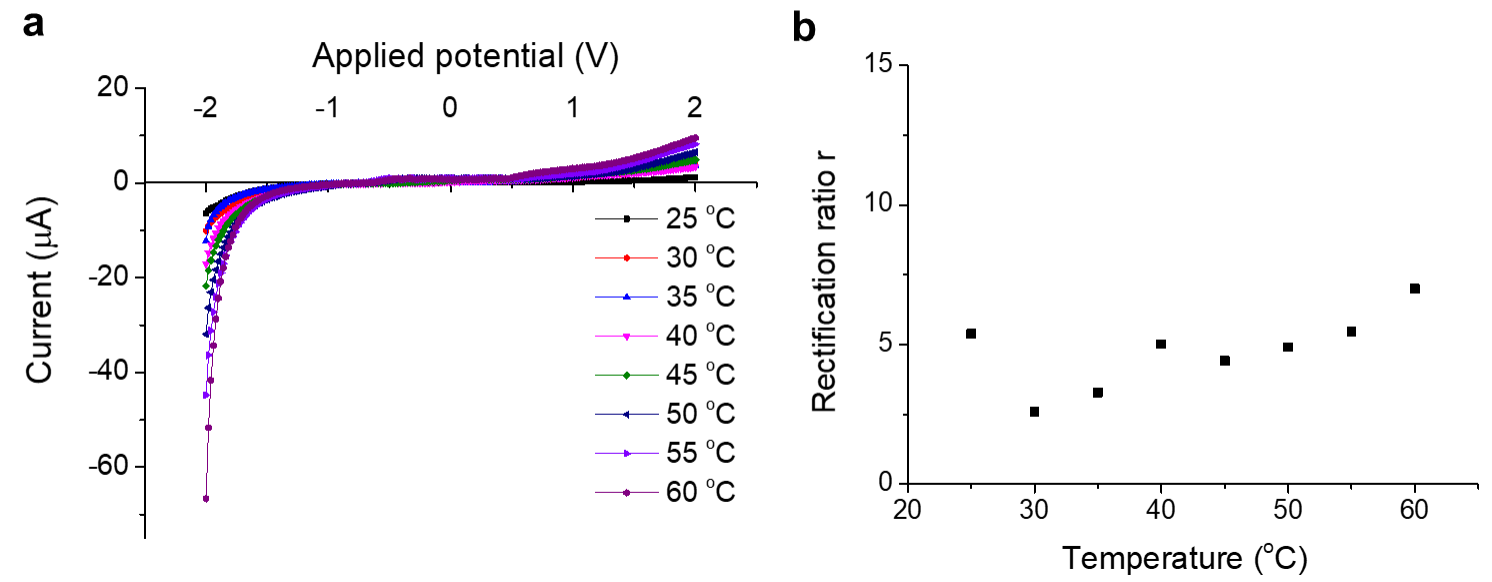

Fig. S6. (a) Current-voltage characteristics of MUA JNPs droplets working at temperatures ranging from $25^{\circ} \mathrm{C}$ to $60{ }^{\circ} \mathrm{C}$. Sweep rates were $0.1 \mathrm{~V} / \mathrm{s}$ in all cases. (b) The corresponding rectification ratios $r=\left|I_{-2 \mathrm{~V}} / I_{+2 \mathrm{~V}}\right|$. Note that the rectification ratios do not vary perceptibly even though the magnitudes of currents increase with temperature. The latter is in line with the kinetic theory of conductance (Phys. Chem. Liq. 2006, 2, 153-165; J. Chem. Eng. Data 2012, 57, 2203-2210), which prescribes that temperature dependence of the molar conductance of ions $(\lambda)$ is $\ln \lambda+2 / 3 \ln \rho=-\frac{\Delta \mathrm{H}}{\mathrm{RT}}+\mathrm{B}$, where $\Delta \mathrm{H}$ is the Eyring activation enthalpy for ion transport, $\rho$ is the density of the liquid, and $\mathrm{B}$ is an integration 
constant. It follows that the molar conductance of ions increases with increasing temperature. Also, note that for the particular droplet tested here, the rectification ratio is $\sim 5$ rather than $\sim 10$ in Fig. S7-S9 - such variability $(\sim 5-15)$ is indeed common in our experiments and may reflect differences in JNP layer quality on different droplets and the quality of contact between the JNPs and the outer electrode.

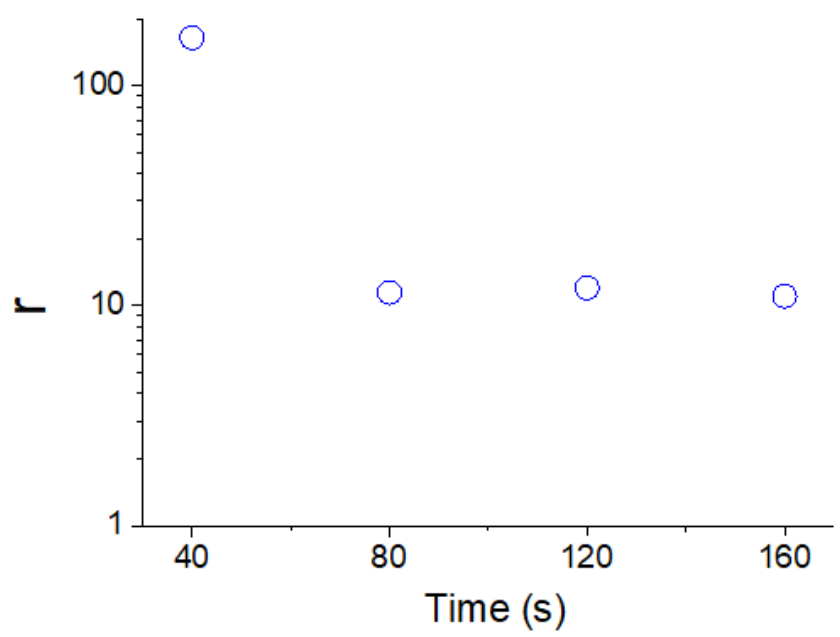

Fig. S7. Rectification ratio of a typical MPA JNP droplet upon cycling the bias between $+2 \mathrm{~V} /-2 \mathrm{~V}$ at $40 \mathrm{~s}$ intervals.

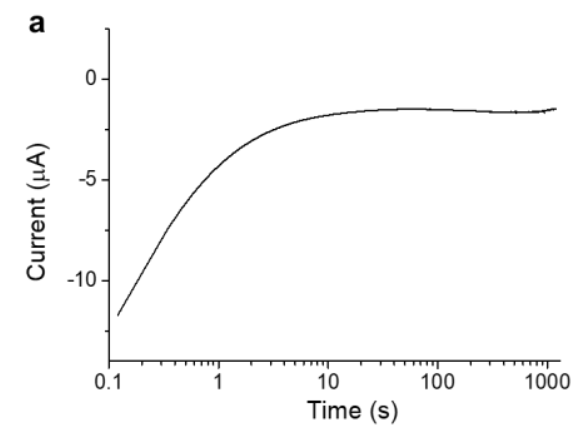

c

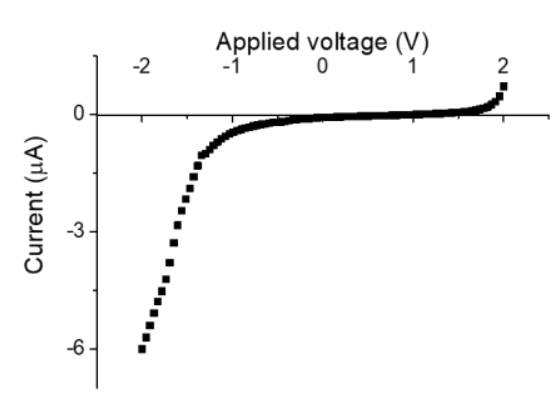

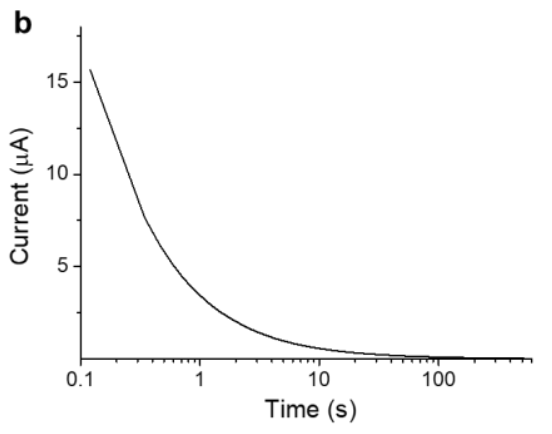

d

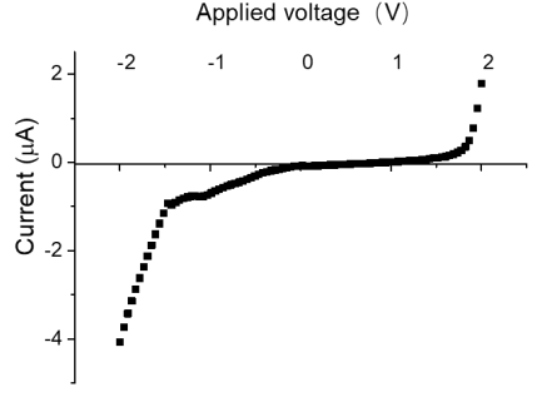

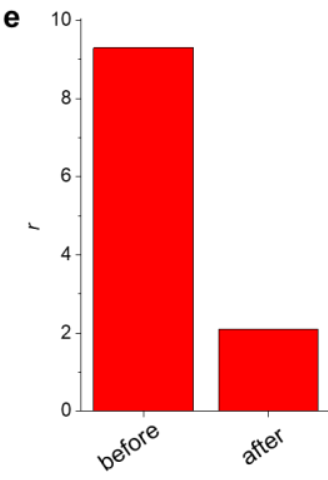

Fig. S8. Stability test of a droplet diode coated with JNPs functionalized with MUA ligands. Current as a function of time (a) under -2.0 V bias applied for $1080 \mathrm{~s}$; and (b) later on, when the bias was changed to $+2.0 \mathrm{~V}$ and applied for another $108 \mathrm{~s}$. In (a), the 
current, after an initial drop (due to double-layer formation), stabilizes at few $\mu \mathrm{A}$; in (b), it gradually decays to only tens of nA. Droplet's current-voltage characteristics (c) before applying a bias voltage. and (d) after applying $-2.0 \mathrm{~V}$ for $1080 \mathrm{~s}$ and then +2.0 $\mathrm{V}$ and $108 \mathrm{~s}$. (e) Rectification ratios, $r=\left|I_{-2 \mathrm{~V}} / I_{+2 \mathrm{~V}}\right|$ calculated from data in (d) and (e). Sweep rates were $0.1 \mathrm{~V} / \mathrm{s}$ in both (c) and (d). The observed decrease in droplet's rectifying performance can be reasonably attributed to fluctuations on the JNP shell, rupture of the JNP membrane due to the hydrogen gas bubbling (see Supporting Movie 1) over a long time, and changes in electrolyte concentration.
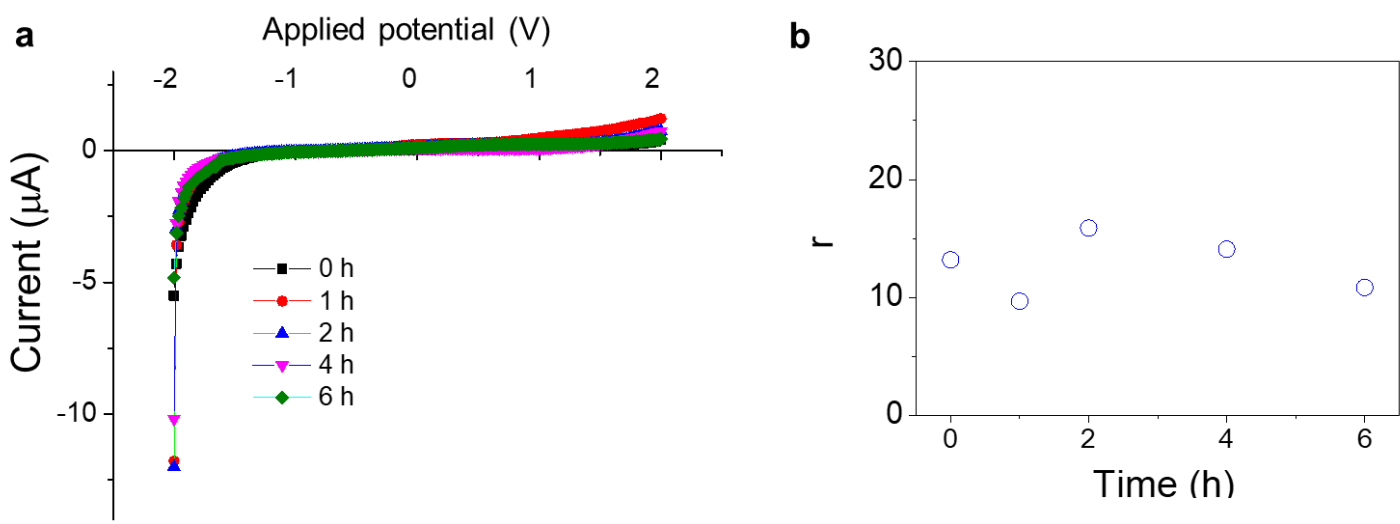

Fig. S9. (a) Current-voltage characteristics of MUA-JNPs droplets recorded after they were submerged - in the absence of prolonged application of bias - in the organic phase for the times indicated in the legend. Sweep rates were $0.1 \mathrm{~V} / \mathrm{s}$ in all cases. Corresponding rectification ratios, $=\left|I_{-2 \mathrm{~V}} / I_{+2 \mathrm{~V}}\right|$, are plotted in (b).

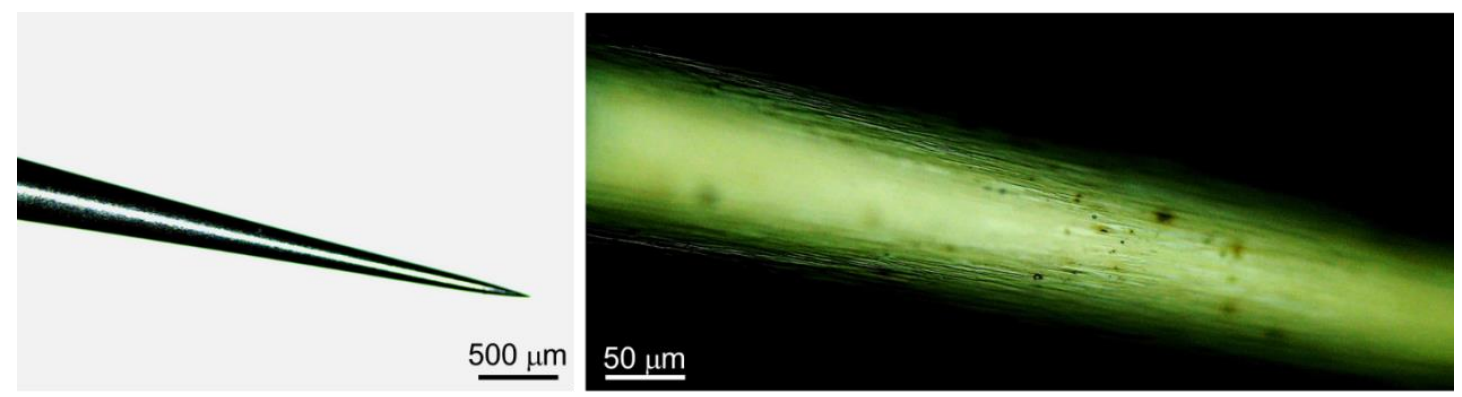

Fig. S10. Optical microscopy images of the outer electrode after five cycles of penetration and extraction. There is no apparent damage of the membrane and no apparent adsorption of the membrane onto the electrode. 


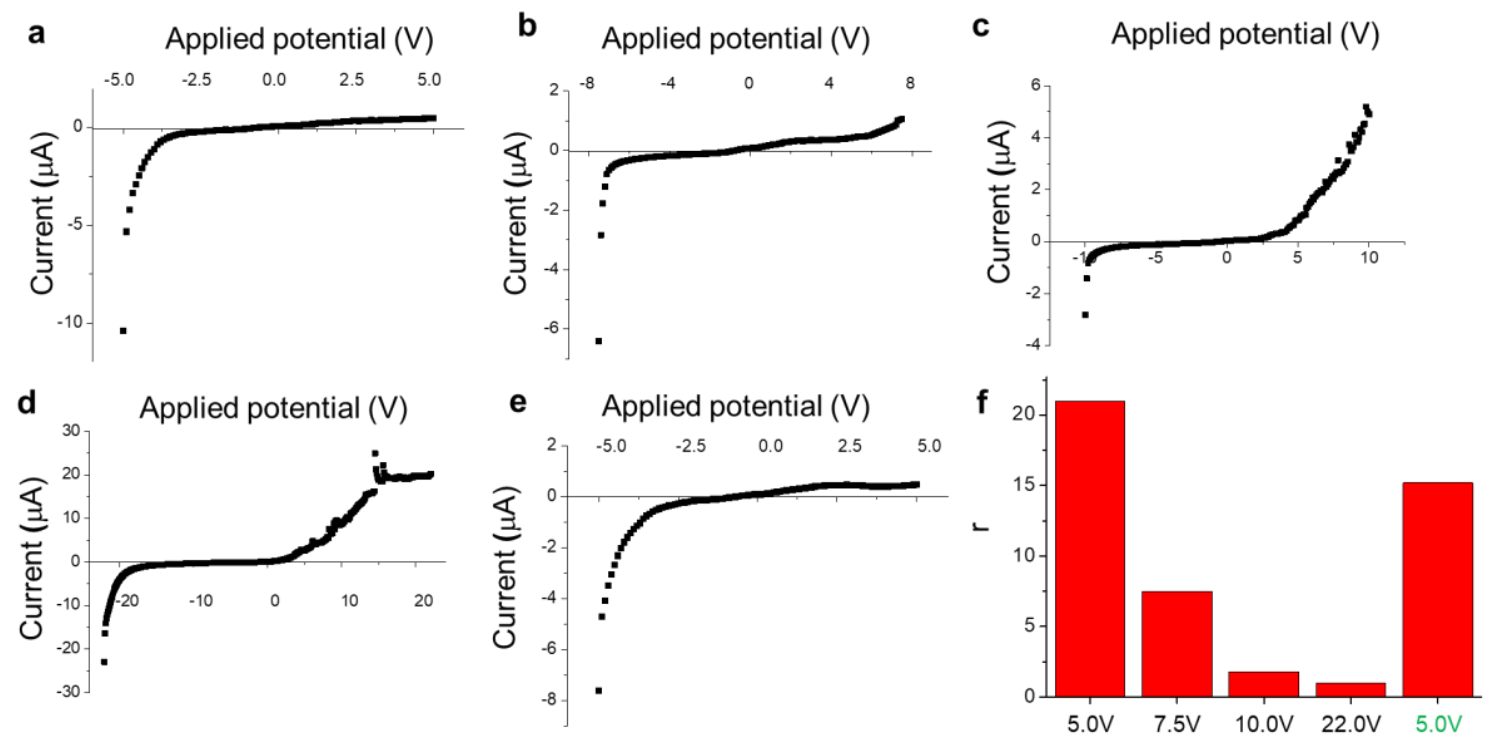

Figure S11. Current-voltage characteristics of MUA JNPs droplets under different ranges of applied bias: (a) $\pm 5.0 \mathrm{~V}$; (b) $\pm 7.5 \mathrm{~V}$; (c) $\pm 10.0 \mathrm{~V}$ and (d) $\pm 22.0 \mathrm{~V}$. In (e), the currentvoltage dependence was recorded between $\pm 5.0 \mathrm{~V}$, after sweeping the bias voltage between $\pm 22.0 \mathrm{~V}$. In all cases, sweep rates were $0.1 \mathrm{~V} / \mathrm{s}$. (f) Rectification ratios $r=$ $\left|I_{-V \max } / I_{+ \text {Vmax }}\right|$ were quantified at the extreme voltages of the ranges in (a-e). As these maximal voltages increase, the rectification ratios decrease. These experiments indicate that the droplets behave as effective diodes only for lower voltages. Comparison between (a) and (e) suggests that the diode membrane may be adversely affected by two processes: 1) Formation of hydrogen bubbles at negative bias voltage $\left(2 \mathrm{H}^{+}+2 \mathrm{e}^{-} \rightarrow \mathrm{H}_{2(\mathrm{~g})}\right)$; 2) Oxidation process observed when the transient surpasses $+5.0 \mathrm{~V}$. The latter is postulated based on prior studies demonstrating oxidation of $\mathrm{Fe}_{3} \mathrm{O}_{4} \mathrm{NPs}$ to a mixture of $\mathrm{Fe}_{3} \mathrm{O}_{4} / \gamma-\mathrm{Fe}_{2} \mathrm{O}_{3}$ domains (see Schwaminger et al. CrystEngComm 2017, 19, 246-255) and may suggest desorption of ligands from the iron-oxide portions of JNPs (overall amounting to the JNP membrane losing its integrity and HER properties). 


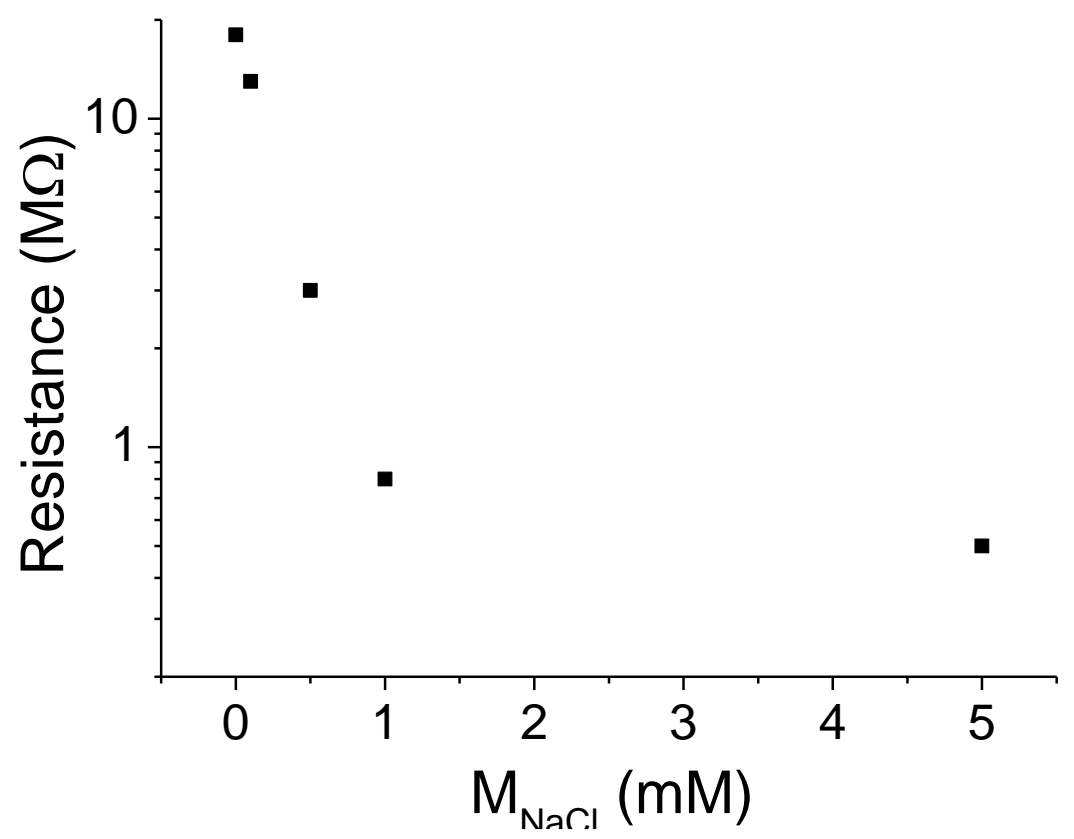

Fig S12. Resistance of a salt-water droplet as a function of $\mathrm{NaCl}$ concentration.

Comment regarding data from Fig. 3. The reader might have noted that the AND gate from Fig. 3 passes a signal at $(0,0)$ state, which merits a further explanation. Please note that in contrast to the OR gate in Fig. $3 \mathrm{a}$, the right terminal of the droplet resistor is maintained at a fixed positive potential $(+3 \mathrm{~V}$, marked as "+V" in the scheme) instead of being grounded as in the OR gate. For this reason, if the potential of both inputs of the AND gate is equal to $V_{\text {in }}$, the output voltage $V_{\text {out }}$ would always be somewhere between the $V_{\text {in }}$ value and $+3 \mathrm{~V}$ maintained at the right terminal of the droplet resistor. More specifically, the value of $V_{\text {out }}$ is defined by the "voltage divider" equivalent scheme (Fig. S13b) containing effective resistances $R_{1}$ and $R_{2}$ of the "diode" droplets and resistance $R$ of the droplet resistor:

$$
\frac{V_{\text {out }}-V_{\text {in }}}{V-V_{\text {in }}}=\frac{\frac{R_{1} R_{2}}{R_{1}+R_{2}}}{R+\frac{R_{1} R_{2}}{R_{1}+R_{2}}}
$$

where $V=+3 \mathrm{~V}$ is the fixed potential on the right terminal of the resistor $R$, and $\frac{R_{1} R_{2}}{R_{1}+R_{2}}$ is the equivalent resistance of two "diode" droplets together. Due to the nonlinear $I-V$ dependence of a single "diode" droplet (see main-text Fig. 1f), the effective resistance of each drop depends on the voltage across the droplet. But $R_{1}=R_{2}$ if potentials of both inputs are equal, because in this case the voltages across each droplet are also equal. If $R_{1}=R_{2}$, the expression $\frac{R_{1} R_{2}}{R_{1}+R_{2}}$ simplifies to $R_{1} / 2$ and $V_{\text {out }}$ 
simplifies to:

$$
V_{\text {out }}=V_{\text {in }}+\frac{R_{1}}{2 R+R_{1}}\left(V-V_{\text {in }}\right)
$$

Data in the main-text Fig. 3b shows that $(0,0)$ inputs $\left(V_{\text {in }}=0 \mathrm{~V}\right)$ results in $V_{\text {out }} \approx$ $0.8 \mathrm{~V}$ and thus $\frac{R_{1}}{2 R+R_{1}} \approx \frac{0.8}{3.0} \approx 0.267$, while the voltage across each "diode" droplet is $-0.8 \mathrm{~V}$. For the $(1,1)$ inputs $\left(V_{\text {in }}=2.0 \mathrm{~V}\right)$, output voltage $V_{\text {out }} \approx 2.5 \mathrm{~V}$, which means that $\frac{R_{1}}{2 R+R_{1}} \approx \frac{2.5-2.0}{3.0-2.0}=0.5$, while the voltage across each "diode" is $-0.5 \mathrm{~V}$. This behavior is consistent with $I-V$ curve in Fig. 1f: the effective resistance $R_{1}$ decreases with a progressively more negative voltage applied across the droplet.
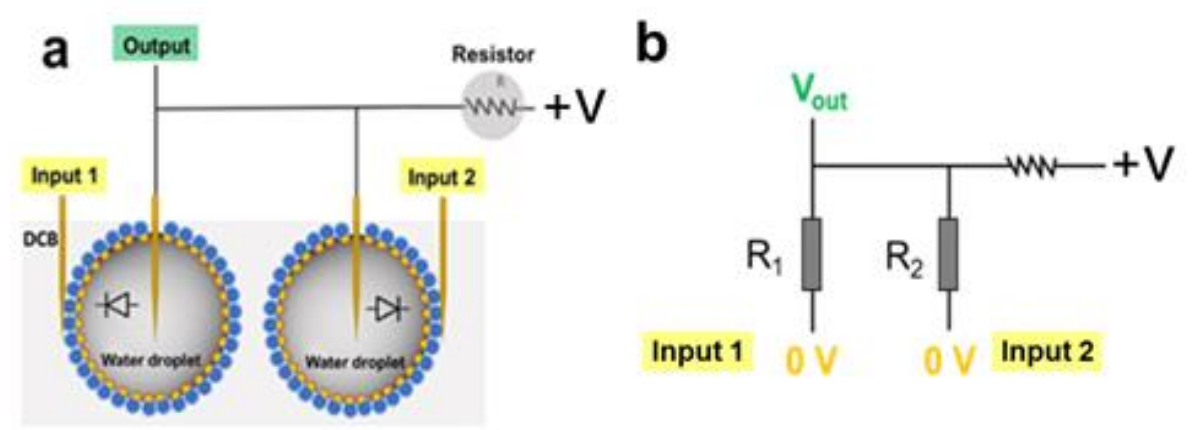

Fig. S13. (a) Scheme of the AND logic gate with droplets. (b) The equivalent circuit of droplet AND gate at some fixed inputs (a case of zero voltage at both inputs is indicated).

\section{Supporting Movie:}

Movie S1. Hydrogen bubble generation at the electrode touching with the JNP membranes. The applied potential is $-2 \mathrm{~V}$. 